\section{Comparaison tarifaire entre la version 1.1 du TARMED et le CPH}

\section{Baumgartner}

\section{Remarques de principe}

Le TARMED constitue, on le sait, un nouveau système tarifaire. Une comparaison directe avec le Catalogue des prestations hospitalières (CPH) n'est, par conséquent, pas possible. Pour une comparaison honnête, il est en effet indispensable de prendre également en considération les conditions-cadres du CPH ainsi que les modalités de facturation du TARMED.

Dans le domaine de l'assurance-maladie, la valeur $\mathrm{du}$ point pour une facturation selon le CPH est de Fr. 4.95. Mais, dans le domaine de l'assurance-maladie, les valeurs du point appliquées sont parfois nettement plus basses. Ainsi, pour les hôpitaux régio-

Correspondance:

Markus Baumgartner

Service tarifaire de la FMH

Case postale 246

CH-6208 Oberkirch naux bernois, la valeur du point est de Fr. 2.90. Par ailleurs, une comparaison tarifaire devrait également considérer les déductions que le CPH prévoit pour les médecins agréés («redevances pour l'infrastructure»), déductions qui peuvent aller jusqu'à 70\%.

Le TARMED tient aussi compte de l'entretien d'information précédant l'intervention et de la prise en charge du patient après celle-ci. Le $\mathrm{CPH}$, quant à lui, ne prévoit pas de position à cet effet. Le consilium, qui a été passablement revalorisé sous TARMED, doit également être pris en considération dans d'éventuelles comparaisons tarifaires. Un consilium d'une durée de 30 minutes donne droit, selon le TARMED, à Fr. 82.74 pour la prestation médicale $(\mathrm{PM})$ et à Fr. 49.14 pour la part technique (PT).

\section{Comparaison entre TARMED 1.1 et $\mathrm{CPH}$}

La comparaison qui suit ne porte, pour le TARMED, que sur la composante médicale (PM) avec une valeur du point de Fr. 1.-. En ce qui concerne le $\mathrm{CPH}$, la valeur du point est de Fr. 4.60, avec une redevance de $30 \%$ pour l'infrastructure.

Les prestations ont été comparées sans l'indemnité d'assistance.

En outre, la durée d'hospitalisation n'est pas prise en compte. A ce sujet, le TARMED prévoit, contrairement au $\mathrm{CPH}$, également une indemnisation pour la prise en charge du patient hospitalisé.

Deutsch erschienen in Nr. 7/2002

Extraction du cristallin

\begin{tabular}{|l|l|l|l|l|l|}
\hline TARMED & \multicolumn{2}{|l|}{ CPH } & \multicolumn{2}{|l|}{} \\
\hline Numéro & Texte & Prix PM & Numéro & Texte & \\
\hline 00.0210 & $\begin{array}{l}\text { Prise en charge du patient hospitalisé, } \\
\text { catégorie A }\end{array}$ & 44.12 & & & \\
\hline 08.2760 & $\begin{array}{l}\text { Extraction du cristallin } \\
\text { ou phaco-émulsification }\end{array}$ & 225.50 & 2784.04 & $\begin{array}{l}\text { Extraction et implantation } \\
\text { d'un cristallin artificiel }\end{array}$ & 1127.- \\
\hline 08.2780 & $\begin{array}{l}\text { + Implantation d'une lentille } \\
\text { artificielle lors d'extraction du cristallin } \\
\text { ou de phaco-émulsification }\end{array}$ & 86.30 & & & \\
\hline Total & & 355.92 & Total & 1127.- \\
\hline
\end{tabular}

Arthroscopie du genou

\begin{tabular}{|l|l|l|l|l|l|}
\hline TARMED & \multicolumn{3}{|l|}{} & \multicolumn{2}{l|}{ CPH } \\
\hline Numéro & Texte & Prix PM & Numéro & Texte & \\
\hline 00.0210 & $\begin{array}{l}\text { Prise en charge du patient hospitalisé, } \\
\text { catégorie A }\end{array}$ & 44.12 & & & \\
\hline 24.5610 & Arthroscopie du genou & 187.52 & 2231.02 & Arthroscopie diagnostique & 161.- \\
\hline Total & & 231.64 & Total & $161 .-$ \\
\hline
\end{tabular}


Traitement chirurgical bilatéral de hernie inguinale

\begin{tabular}{|l|l|l|l|l|l|}
\hline TARMED & \multicolumn{3}{|l|}{ CPH } & Texte \\
\hline Numéro & Texte & Prix PM & Numéro & & \\
\hline 00.0210 & $\begin{array}{l}\text { Prise en charge du patient hospitalisé, } \\
\text { catégorie A }\end{array}$ & 44.12 & & Prix \\
\hline 20.0340 & $\begin{array}{l}\text { Traitement chirurgical bilatéral } \\
\text { de hernie inguinale chez l'adulte, } \\
\text { technique sans tension }\end{array}$ & 612.58 & 2403.00 & Opération de hernie inguinale, & 563.50 \\
\hline Total & & 656.70 & Total & & 536.50 \\
\hline
\end{tabular}

Arthroplastie de la hanche, prothèse totale

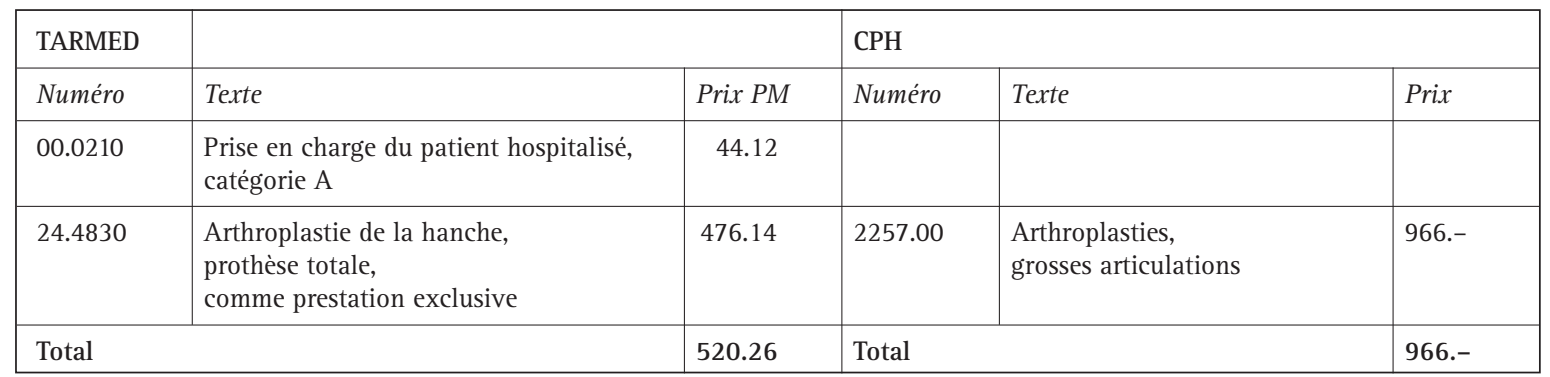

\section{Hernie discale}

\begin{tabular}{|c|c|c|c|c|c|}
\hline \multirow{2}{*}{$\begin{array}{l}\text { TARMED } \\
\text { Numéro }\end{array}$} & \multirow[b]{2}{*}{ Texte } & \multirow[b]{2}{*}{ Prix PM } & \multicolumn{3}{|l|}{$\mathrm{CPH}$} \\
\hline & & & Numéro & Texte & Prix \\
\hline 00.0210 & $\begin{array}{l}\text { Prise en charge du patient hospitalisé, } \\
\text { catégorie A }\end{array}$ & 44.12 & & & \\
\hline 06.0580 & $\begin{array}{l}\text { Fenestration, hémilaminectomie } \\
\text { ou laminotomie unilatérale, } \\
\text { un segment, région lombaire, } \\
\text { procédure neurochirurgicale }\end{array}$ & 168.77 & 2315.00 & $\begin{array}{l}\text { Autres opérations de hernies } \\
\text { discales, sans greffe }\end{array}$ & 805.- \\
\hline 06.0460 & $\begin{array}{l}\text { + Décompression de racine nerveuse } \\
\text { par discectomie, y compris l'ablation } \\
\text { de fragments libres, lors de fenestration, } \\
\text { hémilaminectomie ou laminotomie, } \\
\text { par segment }\end{array}$ & 145.34 & & & \\
\hline 06.0450 & $\begin{array}{l}\text { + Décompression racine nerveuse } \\
\text { par facettotomie et/ou foraminotomie, } \\
\text { lors de fenestration, hémilaminectomie } \\
\text { ou laminotomie, par segment }\end{array}$ & 192.23 & & & \\
\hline \multicolumn{2}{|l|}{ Total } & 550.46 & \multicolumn{2}{|l|}{ Total } & 805.- \\
\hline
\end{tabular}


Appendicectomie

\begin{tabular}{|l|l|l|l|l|l|}
\hline TARMED & \multicolumn{3}{|l|}{ CPH } & Texte & \\
\hline Numéro & Texte & Prix PM & Numéro & \\
\hline 00.0210 & $\begin{array}{l}\text { Prise en charge du patient hospitalisé, } \\
\text { catégorie A }\end{array}$ & 44.12 & & Appendicectomie simple \\
\hline 20.1310 & $\begin{array}{l}\text { Appendicectomie, toute méthode, } \\
\text { comme prestation exclusive }\end{array}$ & 225.03 & 2417.00 & 257.60 \\
\hline Total & & 269.15 & Total & 257.60 \\
\hline
\end{tabular}

\section{Curetage}

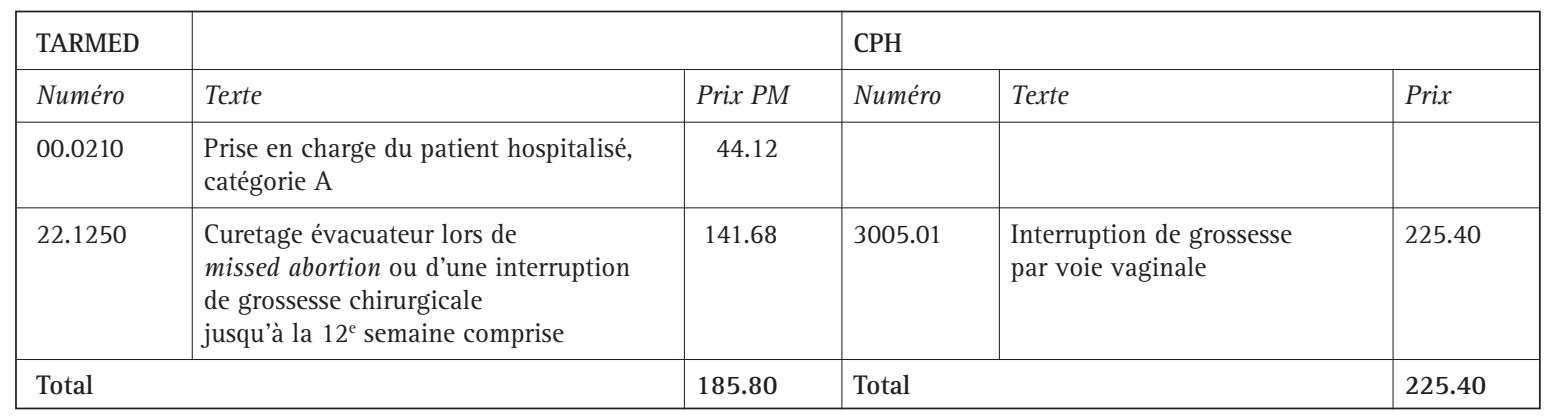

Hystérectomie vaginale

\begin{tabular}{|l|l|l|l|l|l|}
\hline TARMED & \multicolumn{3}{|l|}{ CPH } & \multicolumn{2}{l|}{} \\
\hline Numéro & Texte & Prix PM & Numéro & Texte & \\
\hline 00.0210 & $\begin{array}{l}\text { Prise en charge du patient hospitalisé, } \\
\text { catégorie A }\end{array}$ & 44.12 & & \\
\hline 22.1410 & Hystérectomie vaginale & 450.08 & 3013.00 & Extirpation totale de l'utérus & 805.- \\
\hline Total & & 494.20 & Total & 805.- \\
\hline
\end{tabular}

Tonsillectomie

\begin{tabular}{|l|l|l|l|l|l|}
\hline TARMED & \multicolumn{3}{|l|}{ CPH } & Texte & \\
\hline Numéro & Texte & Prix PM & Numéro & \\
\hline 00.0210 & $\begin{array}{l}\text { Prise en charge du patient hospitalisé, } \\
\text { catégorie A }\end{array}$ & 44.12 & & $\begin{array}{l}\text { Amygdalotomie } \\
\text { selon la méthode de Sluder }\end{array}$ & 225.40 \\
\hline 12.0160 & Tonsillectomie, toute méthode & 183.35 & 2863.00 & & 225.40 \\
\hline
\end{tabular}


Syndrome du tunnel carpien

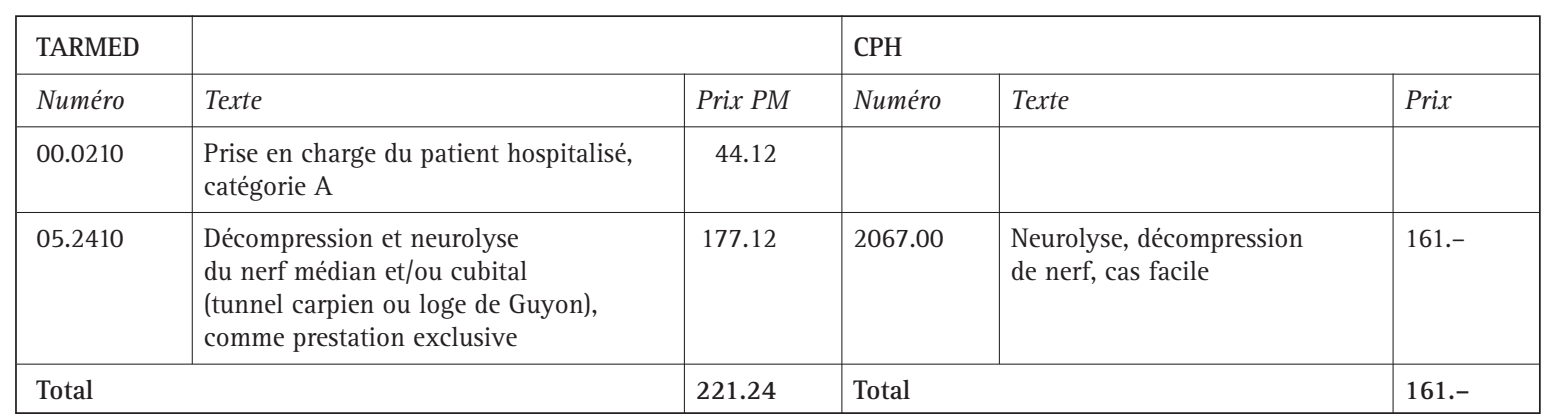

\section{Cure chirurgicale d'hémorroïdes}

\begin{tabular}{|l|l|l|l|l|l|}
\hline TARMED & \multicolumn{3}{|l|}{ CPH } & Texte & \\
\hline Numéro & Texte & Prix PM & Numéro & \\
\hline 00.0210 & $\begin{array}{l}\text { Prise en charge du patient hospitalisé, } \\
\text { catégorie A }\end{array}$ & 44.12 & & Opération d'hémorroïdes \\
\hline 20.2240 & $\begin{array}{l}\text { Cure chirurgicale d'hémorroïdes, } \\
\text { hémorroïdectomie radicale }\end{array}$ & 262.53 & 2445.00 & 193.20 \\
\hline Total & & 306.65 & Total & 193.20 \\
\hline
\end{tabular}

Changement de pile d'un stimulateur définitif

\begin{tabular}{|l|l|l|l|l|l|}
\hline TARMED & \multicolumn{3}{|l|}{ CPH } & Texte & \\
\hline Numéro & Texte & Prix PM & Numéro & & \\
\hline 00.0010 & Consultation, première période de 5 min & 9.57 & 28.71 & & \\
\hline 00.0050 & $\begin{array}{l}\text { Entretien d'information du spécialiste } \\
\text { avec le patient ou ses proches } \\
\text { avant une intervention diagnostique } \\
\text { ou thérapeutique, par période } \\
\text { de 5 min (3 fois / 15 min) }\end{array}$ & $\begin{array}{l}\text { Changement de pile } \\
\text { d'un stimulateur définitif }\end{array}$ & 137.53 & 2382.00 & $\begin{array}{l}\text { Changement de pile } \\
\text { d'un stimulateur du cœur }\end{array}$ \\
\hline 17.1560 & & 175.81 & Total & 128.80 \\
\hline Total & & 128.80 \\
\hline
\end{tabular}

